\title{
Bifurcations in a Generalization of the ZAD Technique: Application to a DC-DC Buck Power Converter
}

\author{
Ludwing Torres, ${ }^{1}$ Gerard Olivar, $^{1}$ and Simeón Casanova $^{2}$ \\ ${ }^{1}$ Percepción y Control Inteligente, Departamento de Ingeniería Eléctrica, \\ Facultad de Ingeniería y Arquitectura, Universidad Nacional de Colombia, Sede Manizales, \\ Electrónica y Computación, Bloque Q, Campus La Nubia, Manizales, Colombia \\ ${ }^{2}$ ABC Dynamics, Departamento de Matemáticas y Estadística, \\ Facultad de Ciencias Exactas y Naturales, Universidad Nacional de Colombia, \\ Sede Manizales, Bloque Y, Campus La Nubia, Manizales, Colombia
}

Correspondence should be addressed to Gerard Olivar, golivart@unal.edu.co

Received 21 March 2012; Accepted 30 April 2012

Academic Editor: Ahmad M. Harb

Copyright (C) 2012 Ludwing Torres et al. This is an open access article distributed under the Creative Commons Attribution License, which permits unrestricted use, distribution, and reproduction in any medium, provided the original work is properly cited.

A variation of ZAD technique is proposed, which is to extend the range of zero averaging of the switching surface (in the classic ZAD it is taken in a sampling period), to a number $K$ of sampling periods. This has led to a technique that has been named $K-Z A D$. Assuming a specific value for $K=2$, we have studied the 2-ZAD technique. The latter has presented better results in terms of stability, regarding the original ZAD technique. These results can be demonstrated in different state space graphs and bifurcation diagrams, which have been calculated based on the analysis done about the behavior of this new strategy.

\section{Introduction}

Currently, power electronics has an important place in industry. This is largely due to the very extensive number of applications derived from these systems, including control of power converters. This was the main motivation for researchers worldwide to develop new advances in this field and has promoted the investigation on many mathematical models. They correspond usually to variable structure systems, chaos, and control. The practical goal is to obtain better devices or new and improved mechanisms for use in electronic controllers.

Bifurcation, chaos, and control in electronic circuits have been reported in many papers such as $[1,2]$. Regarding power electronics, early works can be found in the literature from the 1980s when first observed in [3, 4]. In 1989 several authors such as Krein and Bass [5], and 
Deane and Hamill [6] began to study chaos in various power electronic circuits. In his work Wood [7] contributed to the understanding of chaos, showing in phase diagrams that the pattern of the trajectories was messy as small variation on initial conditions and parameters in the system were performed. Meanwhile Deane and Hamill reported some of the best works on bifurcations and chaos applied to electronic circuits. Their studies were based on computer simulations and laboratory experiments $[3,4,6]$.

Because of the multiple side effects that generation of chaos caused in these systems, several authors proceeded to develop different control techniques, such as [8] by Ott et al., who found a way of controlling unstable orbits coexisting with chaos. They used small disturbances. This resulted in the so well-known OGY method (after the names of the authors Ott, Grebogi, and Yorke). Pyragas [9] contributed to this topic with a feedback scheme using a time-delayed control known as (time-delayed autosynchronization) TDAS.

Also the work by Utkin is very important in the control literature. He studied (pulsewidth modulation) PWM systems and variable structure systems [10]. He introduced a now very popular control technique so-called sliding-mode control (SMC) and many applications were proposed [11]. These studies were taken later as a starting point by Carpita et al. [12]. In his work, Carpita presents a sliding mode controller for a (uninterrupted power system) UPS. Carpita uses a sliding surface which is a linear combination of error variable and its derivative.

Some time after, a new technique, both conceptually different from sliding-mode control (although very related to it) and as a practical implementation to SMC, appeared. It was called (zero average dynamics) ZAD by the authors. This control technique forces the system to have, at each clock cycle, a zero average of the sliding surface (instead to be zero all the time, as in sliding-mode control). Application of this technique to a buck converter with centered pulse and an approximation scheme needed for the practical implementations [12] has obtained very good performance. Robustness, low stationary error, and fixed switching frequency have also been achieved [13]. It has been observed that as the main parameter in the surface $k_{s}$ is decreased, chaotic behavior appears which is not desirable in practice. Then, several additional techniques such as TDAS (mentioned before) or (fixed point induced control) FPIC must be implemented in order to guarantee a wider operation range [13]. FPIC has obtained the best results with regards to stabilization and chaos control. Bifurcation diagrams have shown flip or period-doubling bifurcations followed by border-collision bifurcations due to the saturation of the limit cycle. This sequence of bifurcations leads the system to chaotic operation for low values of $k_{s}$. When saturation of the limit cycle appears, the $\mathrm{ZAD}$ technique is degraded and thus zero average in each cycle is lost.

In order to solve this problem, we generalize the ZAD technique (from now on called classical ZAD) to the so-called K-ZAD technique. This generalization allows the surface to be of zero average not in every cycle (as classical ZAD), but in a sequence of $K$ cycles. Being a weaker condition one can choose the duty cycles in such a way that they are not saturated (at least not so frequently) and thus zero average and stabilization in a periodic orbit is obtained.

Specifically, our study has focused on the value $K=2$, which gives rise to 2-ZAD, that is, two sampling periods for the zero average in the surface. We compare this generalization with the classical ZAD through bifurcation diagrams.

The remaining of this paper is organized as follows. In Section 2 we explain in detail mathematical modelling of this technique and we perform the corresponding algebraic computations. In Section 3 the numerical implementation and the results are discussed. Finally, conclusions and future work are stated in Section 4. 


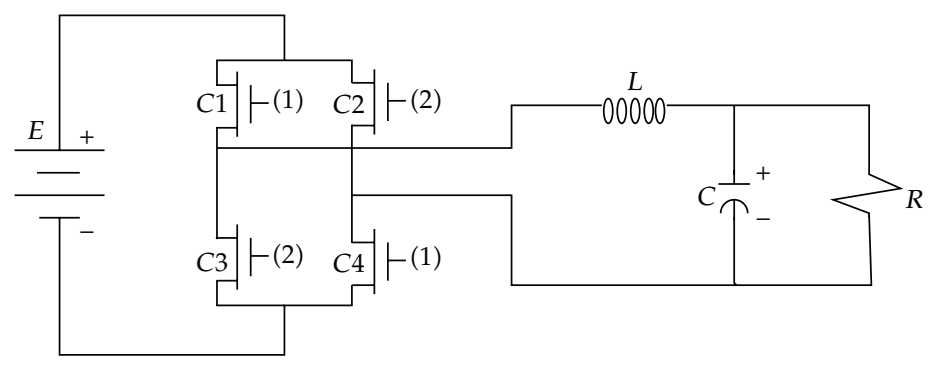

Figure 1: Scheme of the Buck converter.

\section{2. $K$-ZAD Strategy}

The scheme for the buck converter is shown in Figure 1. We donot take into account the diode full dynamics and thus we assume always continuous conduction mode (alternatively, we can consider bidirectional switches which allow negative currents).

The classical diode-transistor scheme is changed by a transistors bridge linked with the source. When transistors are in (1) position the pulse magnitude is $+E$, and when the position is (2.2), then the magnitude is $-E$. As a result we have a PWM system which inverts the polarity at each switching.

With the aim of obtaining a nondimensional system with nondimensional parameters, which leads to easier analysis, we perform the following change of variables [13]:

$$
x_{1}=\frac{v}{E}, \quad x_{1 \mathrm{ref}}=\frac{V_{\mathrm{ref}}}{E}, \quad x_{2}=\frac{1}{V} \sqrt{\frac{L}{C}} i, \quad r=\frac{1}{R} \sqrt{\frac{L}{C}} .
$$

Also we normalize the sampling period

$$
T=\frac{T_{c}}{\sqrt{L C}}
$$

where $T_{c}=50 \mu \mathrm{s}, R=20 \Omega, C=40 \mu \mathrm{F}, L=2 \mathrm{mH}$, and $E=40 \mathrm{~V}$. These values come from laboratory prototypes which can be found in the literature [13]. Thus we get the value $T=$ 0.1767 and the following nondimensional system:

$$
\left(\begin{array}{c}
\dot{x}_{1} \\
\dot{x}_{2}
\end{array}\right)=\left(\begin{array}{ll}
-\gamma & 1 \\
-1 & 0
\end{array}\right)\left(\begin{array}{l}
x_{1} \\
x_{2}
\end{array}\right)+\left(\begin{array}{l}
0 \\
1
\end{array}\right) u
$$

which can be written in compact form as $\dot{x}=A x+b u$ where

$$
A=\left(\begin{array}{ll}
-\gamma & 1 \\
-1 & 0
\end{array}\right), \quad b=\left(\begin{array}{l}
0 \\
1
\end{array}\right), \quad \dot{x}=\left(\begin{array}{c}
\dot{x}_{1} \\
\dot{x}_{2}
\end{array}\right), \quad x=\left(\begin{array}{c}
x_{1} \\
x_{2}
\end{array}\right)
$$


The solution of this piecewise-linear system can be computed algebraically and the expression for the Poincaré map corresponding to sampling every $T$ time units is [14]

$$
x((k+1) T)=e^{A T} x(k T)+\left(e^{A\left(T-d_{k} / 2\right)}+I\right) A^{-1}\left(e^{A\left(d_{k} / 2\right)}-I\right) b-e^{A\left(d_{k} / 2\right)} A^{-1}\left(e^{A\left(T-d_{k}\right)}-I\right) b .
$$

$u$ stands for the control action, which corresponds to a centered pulse, which can be mathematically written as

$$
u= \begin{cases}1, & \text { if } k T<t<k T+\frac{d}{2} \\ -1, & \text { if } k T+\frac{d}{2}<t<k T+\left(T-\frac{d}{2}\right) \\ 1, & \text { if } k T+\left(T-\frac{d}{2}\right)<t<k T+T\end{cases}
$$

$d$ corresponds to the so-called duty cycle. It will be computed in such a way that the dynamical system tends to the sliding surface defined as

$$
s(x(t))=\left(x_{1}-x_{1 \mathrm{ref}}\right)+k_{s}\left(\dot{x}_{1}-\dot{x}_{1 \mathrm{ref}}\right) .
$$

The ZAD technique imposes that the orbit must be such that when the variables are replaced in the sliding surface in (2.7), the following equation is fulfilled:

$$
\int_{k T}^{(k+1) T} s(x(t)) d t=0 .
$$

Computing $d$ from (2.8) involves transcendental expressions which should be avoided in practical applications. Thus in the literature, the sliding surface has been approximated by a piecewise-linear function. This allows an algebraic solution for $d$, which is used in applications.

\subsection{Computations for the Classical ZAD Strategy}

Figure 2 shows how the classical ZAD strategy works. The area under the curve $s(x)$ must be zero. 


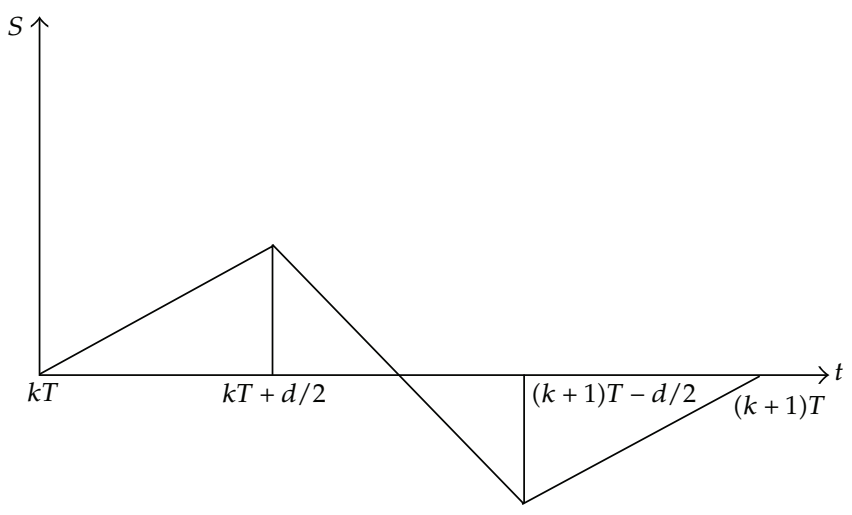

Figure 2: Scheme for the classical ZAD strategy.

Using the piecewise-linear approximation, the integral can be written as

$$
\begin{aligned}
\int_{k T}^{(k+1) T} s(x(t)) d t \approx & \int_{k T}^{k T+d / 2}\left(s(x(k T))+(t-k T) \dot{s}_{1}(x(k T)) d t\right. \\
& +\int_{k T+d / 2}^{(k+1) T-d / 2}\left(s(x(k T))+\frac{d}{2} \dot{s}_{1}(x(k T))+\left(t-\left(k T+\frac{d}{2}\right)\right) \dot{s}_{2}(x(k T))\right) d t \\
& +\int_{(k+1) T-d / 2}^{(k+1) T} s(x(k T))+(T-d) \dot{s}_{2}(x(k T))+(t-(k+1) T+d) \dot{s}_{1}(x(k T)) d t,
\end{aligned}
$$

where $s(x(k T))$ is the value of the sliding surface at the sampling instant, $\dot{s}_{1}(x(k T))$ is the derivative in the first and third pieces of the piecewise-linear approximation and $\dot{s}_{2}(x(k T))$ is the derivative in the central piece. After some algebra we get

$$
d=\frac{2 s(x(k T))+T \dot{s}_{2}(x(k T))}{\dot{s}_{2}(x(k T))-\dot{s}_{1}(x(k T))} .
$$

Since a value $d>T$ or $d<0$ is practically impossible, when this occurs the duty cycle is saturated in such a way that we take 0 if $d \leq 0$ and we take $T$ if $d \geq T$. In those cases where $d$ is saturated the ZAD strategy fails and thus the zero average condition is not fulfilled.

\subsection{Computations for the K-ZAD Strategy}

Now we consider the computations when the zero average condition is weakened to a sequence of $K$ cycles instead of every cycle. Previously, with the classical ZAD strategy we had

$$
\int_{T}^{(k+1) T} s(x(t)) d t=0 .
$$




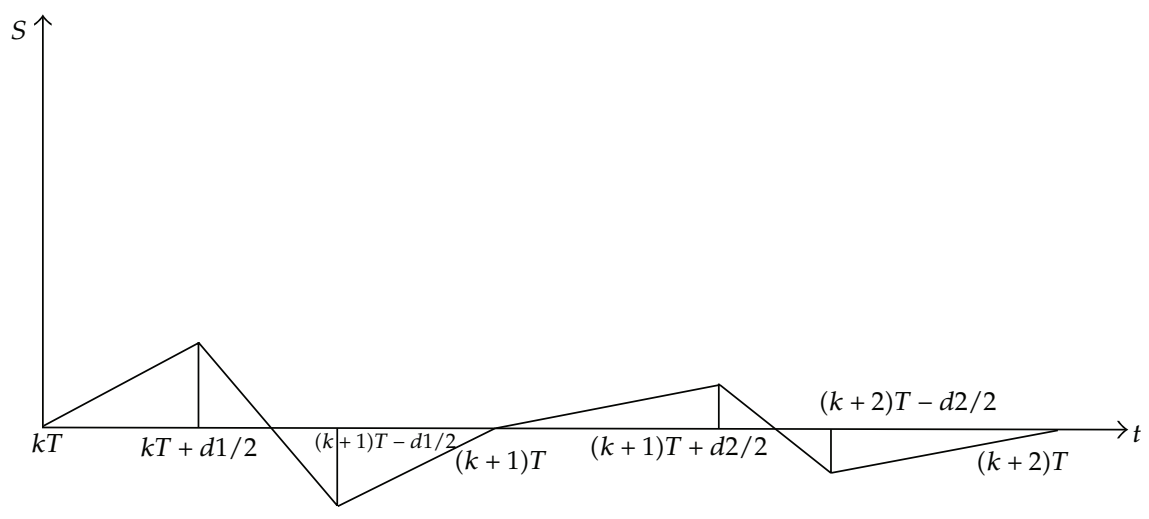

Figure 3: Scheme for the 2-ZAD technique.

Now, with the $K-Z A D$ strategy, we have

$$
\int_{k T}^{(k+1) T} s(x(t)) d t+\int_{(k+1) T}^{(k+2) T} s(x(t)) d t+\cdots+\int_{(k+(K-1)) T}^{(k+K) T} s(x(t)) d t=0 .
$$

This means that we extend the condition of zero average to an interval of $K$ consecutive cycles.

\subsection{Computations for the 2-ZAD Strategy}

In the case $K=2$ we get

$$
\int_{k T}^{(k+1) T} s(x(t)) d t+\int_{(k+1) T}^{(k+2) T} s(x(t)) d t=0
$$

In Figure 3 we depict a scheme for the 2-ZAD. The area under the curve between $t=k T$ and $t=(k+2) T$ must be zero.

Since we are now dealing with two cycles, we can have two different duty cycles and different values for the derivatives of the piecewise-linear functions. Thus we introduce the following notation.

(i) In the first cycle we write $d_{1}$ for the duty cycle and we write $d_{2}$ for the duty cycle in the second cycle.

(ii) For the derivatives we use $\dot{s}_{11}$ and $\dot{s}_{21}$ for the first cycle. They correspond to $\dot{s}_{1}(x(k T))$ and $\dot{s}_{2}(x(k T))$ for the first cycle. They are computed according to the sign of $u$ and are sampled at the beginning of the first interval (i.e., at $t=k T$ ). In the second interval we will denote the derivatives by $\dot{s}_{12}$ and $\dot{s}_{22}$. They correspond to $\dot{s}_{1}(x((k+1) T))$ and $\dot{s}_{2}(x((k+1) T))$ for the second interval. They are computed sampling at the beginning of the second cycle (i.e., at $t=(k+1) T)$ as it is shown in Figure 3. 
With this notation and some further algebra, we get

$$
s=\dot{s}_{11}(t-k T)+s(x(k T)) .
$$

For the second piece of the first cycle $t \in\left(k T+d_{1} / 2,(k+1) T-d_{1} / 2\right)$, with derivative $\dot{s}_{21}(x(k T))$ we get

$$
s=\dot{s}_{21}\left(t-k T-\frac{d_{1}}{2}\right)+\dot{s}_{11}\left(\frac{d_{1}}{2}\right)+s(x(k T)) .
$$

For the third piece $t \in\left((k+1) T-d_{1} / 2,(k+1) T\right)$ of the first interval, with derivative $\dot{s}_{11}(x(k T))$, we get

$$
s=\dot{s}_{11}\left(t-(k+1) T+d_{1}\right)+\dot{s}_{21}\left(T-d_{1}\right)+s(x(k T)) .
$$

For the second cycle $t \in((k+1) T,(k+2) T)$ we have derivatives $\dot{s}_{12}$ and $\dot{s}_{22}$ and the duty cycle $d_{2}$. In the first piece of the second cycle $t \in\left((k+1) T,(k+1) T+d_{2} / 2\right)$, with derivative $\dot{s}_{12}(x((k+1) T))$, we get

$$
s=\dot{s}_{12}(t-(k+1) T)+\dot{s}_{11}\left(d_{1}\right)+\dot{s}_{21}\left(T-d_{1}\right)+s(x(k T)) .
$$

For the second piece in the second cycle $t \in\left((k+1) T+d_{2} / 2,(k+2) T-d_{2} / 2\right)$, with derivative $\dot{s}_{22}(x((k+1) T))$, we get

$$
s=\dot{s}_{22}\left(t-(k+1) T-\frac{d_{2}}{2}\right)+\dot{s}_{12}\left(\frac{d_{2}}{2}\right)+\dot{s}_{11}\left(d_{1}\right)+\dot{s}_{21}\left(T-d_{1}\right)+s(x(k T)) .
$$

Finally, for the third piece in the second interval $t \in\left((k+2) T-d_{2} / 2,(k+2) T\right)$, with derivative $\dot{s}_{12}(x((k+1) T))$, we get

$$
s=\dot{s}_{12}\left(t-(k+2) T+d_{2}\right)+s_{22}\left(T-d_{2}\right)+\dot{s}_{11}\left(d_{1}\right)+\dot{s}_{21}\left(T-d_{1}\right)+s(x(k T)) .
$$

Thus we have

$$
\begin{aligned}
& \int_{0}^{T} s(x(t)) d t+\int_{T}^{2 T} s(x(t)) d t \approx \int_{k T}^{k T+d_{1} / 2} \dot{s}_{11}(t-k T)+s(x(k T)) d t \\
& \quad+\int_{k T+d_{1} / 2}^{(k+1) T-d_{1} / 2} \dot{s}_{21}\left(t-k T-\frac{d_{1}}{2}\right)+\dot{s}_{11}\left(\frac{d_{1}}{2}\right)+s(x(k T)) d t \\
& \quad+\int_{(k+1) T-d_{1} / 2}^{(k+1) T} \dot{s}_{11}\left(t-(k+1) T+d_{1}\right)+\dot{s}_{21}\left(T-d_{1}\right)+s(x(k T)) d t \\
& +\int_{(k+1) T}^{(k+1) T+d_{2} / 2} \dot{s}_{12}(t-(k+1) T)+\dot{s}_{11}\left(d_{1}\right)+\dot{s}_{21}\left(T-d_{1}\right)+s(x(k T)) d t
\end{aligned}
$$




$$
\begin{aligned}
& \int_{(k+1) T+d_{2} / 2}^{(k+2) T-d_{2} / 2} \dot{s}_{22}\left(t-(k+1) T-\frac{d_{2}}{2}\right)+\dot{s}_{12}\left(\frac{d_{2}}{2}\right) \\
& \quad+\dot{s}_{11}\left(d_{1}\right)+\dot{s}_{21}\left(T-d_{1}\right)+s(x(k T)) d t \\
& \quad+\int_{(k+2) T-d_{2} / 2}^{(k+2) T} \dot{s}_{12}\left(t-(k+2) T+d_{2}\right)+s_{22}\left(T-d_{2}\right)+\dot{s}_{11}\left(d_{1}\right) \\
& \quad+\dot{s}_{21}\left(T-d_{1}\right)+s(x(k T)) d t .
\end{aligned}
$$

Solving the integral we have the following expression for $d_{2}$ as a function of $d_{1}$ :

$$
d_{2}=\frac{-\left(3 d_{1} \dot{s}_{11}+3 T \dot{s}_{21}+4 s(x(k T))+T \dot{s}_{22}-3 d_{1} \dot{s}_{21}\right)}{\left(\dot{s}_{12}-\dot{s}_{22}\right)}
$$

Since we have $d_{1}$ as an independent variable and $d_{2}$ is dependant of $d_{1}$, we can assume that $d_{1}$ is nonsaturated. Then our proposal is to choose $d_{2}$ to be as the optimal value (in a certain sense) taking $d_{1}$ between 0 and T. Our optimal condition will be choosing $d_{2}$ the closest to the stationary theoretical value as possible. In this way we approximate $x_{1}$ to the desired regulation value as much as possible.

\section{Numerical Results}

Following [13] the stationary theoretical value for the duty cycle was computed as

$$
d_{\mathrm{eq}}=T \frac{1+x_{1 \mathrm{ref}}}{2} .
$$

Then according to the previous section, the following results are obtained.

\subsection{Results}

In the literature it was found that, for the classical ZAD strategy and centered pulse, stability of the $T$-periodic orbit was obtained in the range $k_{s}>3.23$, approximately. Below this value, period doubling and border collision bifurcation due to saturations lead the system to big amplitude chaotic operation, which is inadmissible in practical devices. The ranges considered for the 2-ZAD strategy contain values from $k_{s}=0.01$ to $k_{s}=5$. We can observe the transition from stability to chaos.

Figure 4 shows bifurcations obtained for the classical ZAD strategy, for parameter values less than $k_{s}=3.23$. For parameter values greater than 3.23 , the $T$-periodic orbit is stable. The duty cycle is approximately 0.1590 when $x_{1 \text { ref }}=0.8$ and $\gamma=0.35$. In the figures we observe that chaos is obtained when $k_{s}$ decreases. For parameter values $k_{s}$ less than 1 , big amplitude chaos appears. The regulation error also grows. Close to $k_{s}=3.23$ we can also observe a fast transition of the stable $2 T$-periodic orbit into saturation.

In the computations corresponding to Figure 5 we considered 2-ZAD strategy all the time, for all values of parameter $k_{s}$. We allowed saturations when it was impossible to get 


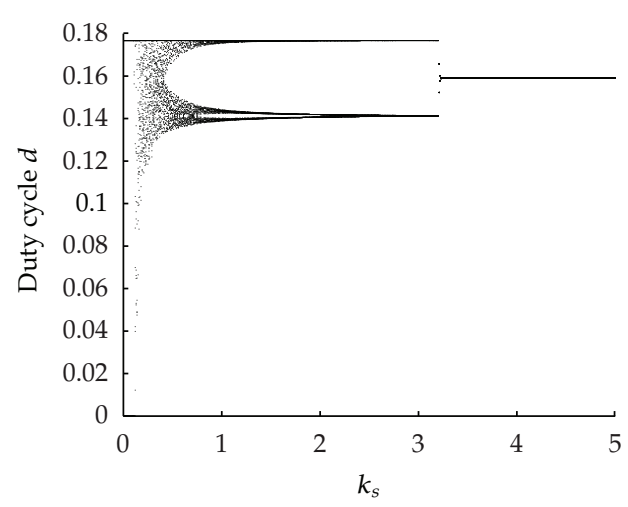

(a) Bifurcations in the duty cycle

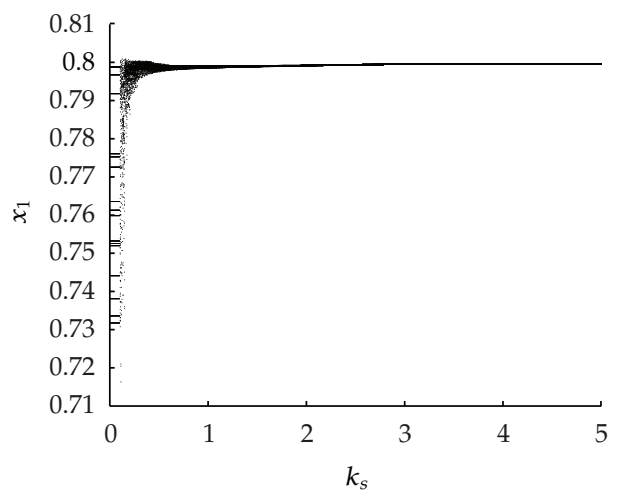

(c) Bifurcations associated with the nondimensional voltage in the capacitor

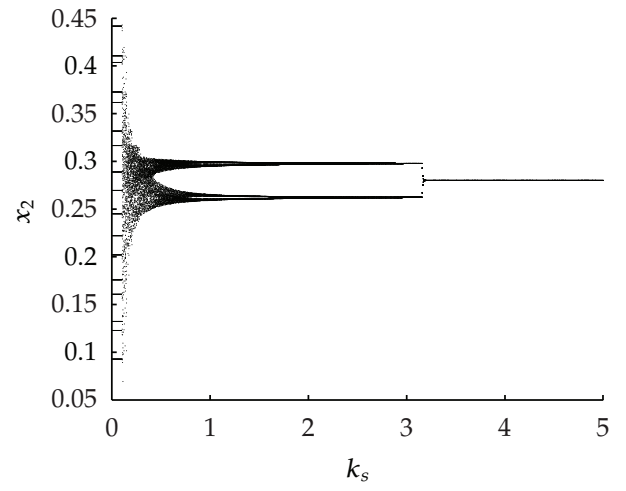

(b) Bifurcations associated with the nondimensional current in the inductor

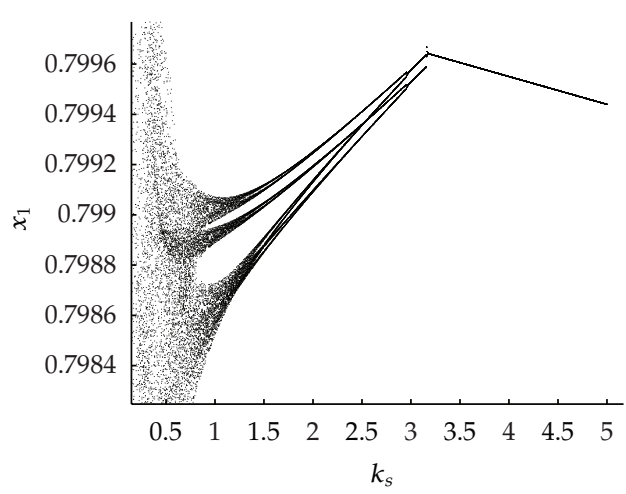

(d) Zoom of the bifurcation diagram associated with the nondimensional voltage

Figure 4: Bifurcation diagram with bifurcation parameter $k_{s}$, in the classical ZAD strategy.

two nonsaturated duty cycles. In these figures it can be observed that the stability range of the $T$-periodic orbit is wider than that for the classical ZAD strategy. From $k_{s}=5$ until approximately $k_{s}=0.5$ the 2-ZAD strategy avoids the orbit to get far from the stable $T$ periodic orbit. This is mainly due to the existence of nonsaturated duty cycles.

Figures $6,7,8$, and 9 correspond to a reference value 0.8 and initial conditions $x_{1}=x_{1 \text { ref }}$ and $x_{2}=\gamma x_{1 \text { ref }}$ (stable state values according to [13]). Initial conditions close to the stable state value are considered in order to check the performance of these techniques. With the aim of getting a better insight of the behavior of the orbit in stable state, we take the last values of the trajectory. Thus we compare both the classical ZAD strategy with 2-ZAD. Results show that 2-ZAD performs much better than classical ZAD technique.

Regarding chaotic behavior, Figure 10 shows the Lyapunov exponents for both techniques. It can be observed that in the 2-ZAD case the exponents are below zero for a wider range than for the classical ZAD strategy. For the 2-ZAD, stability is kept almost until $k_{s}$ close to 0.7 . Then some small amplitude period-doubling bifurcations appear. Close to $k_{s}=0.25$ and below, saturation in the duty cycle cannot be avoided and chaos appears. 


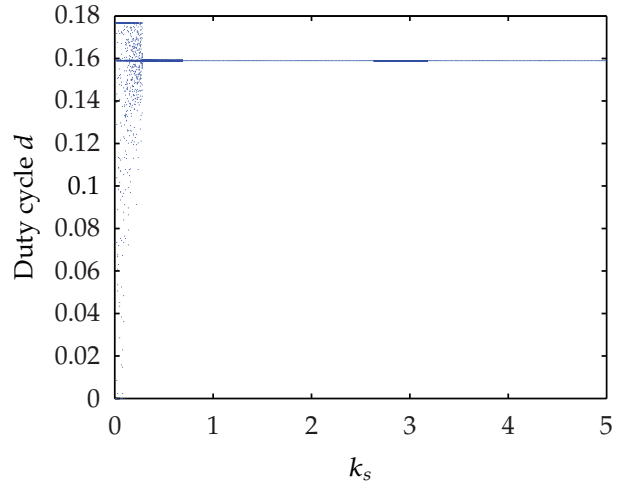

(a) Bifurcations of the duty cycle

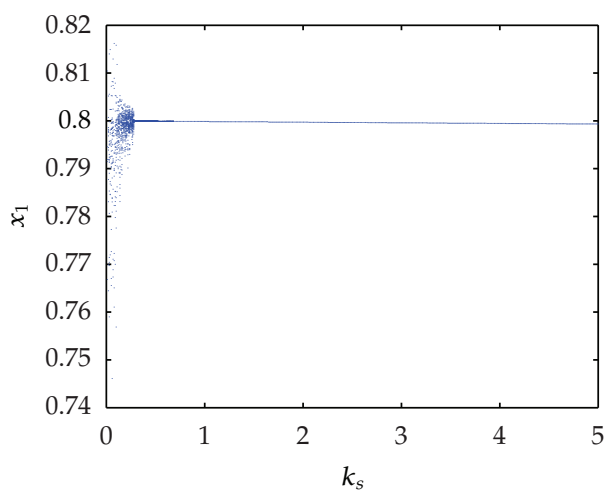

(c) Bifurcations of the variable associated with the voltage in the capacitor

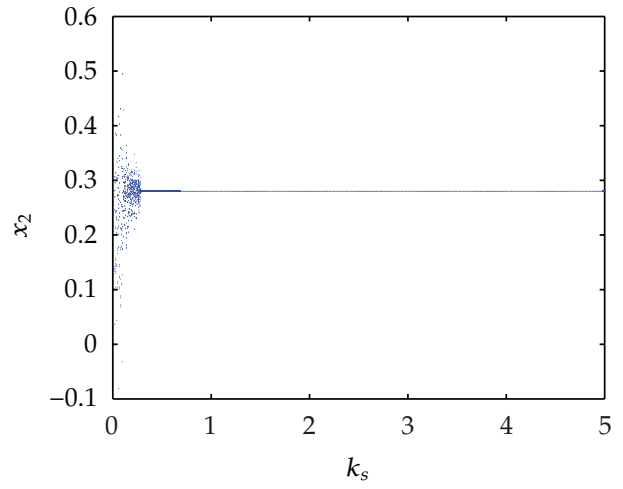

(b) Bifurcations of the variable associated with the current in the inductor

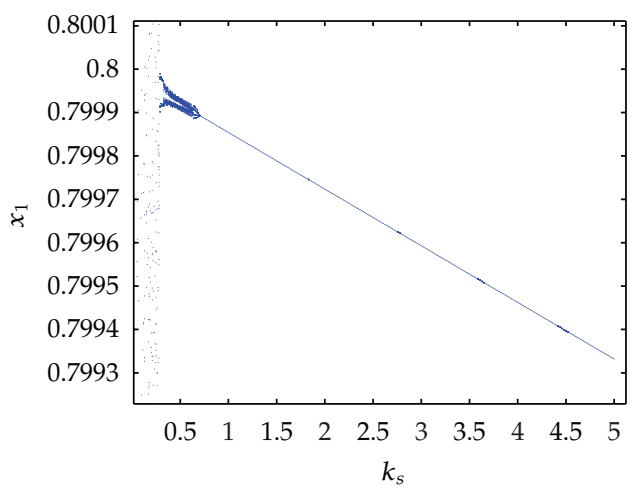

(d) Zoom of the bifurcation diagram corresponding with the variable associated to the voltage

Figure 5: Bifurcation diagrams with bifurcation parameter $k_{s}$ when 2-ZAD strategy is considered.

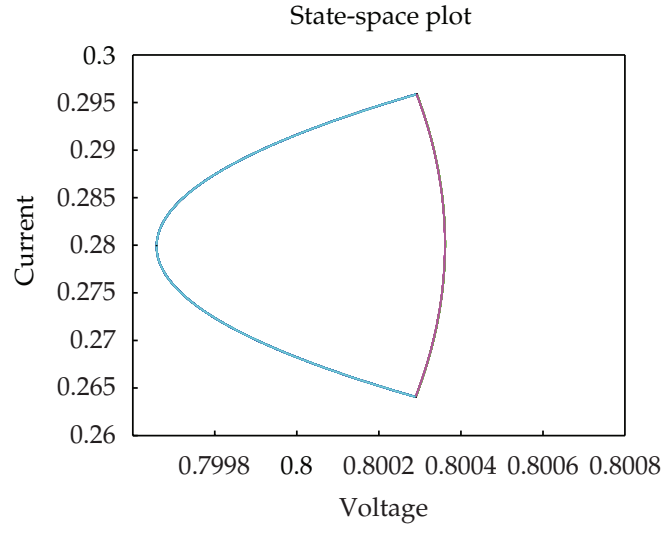

(a) Orbit in the state space

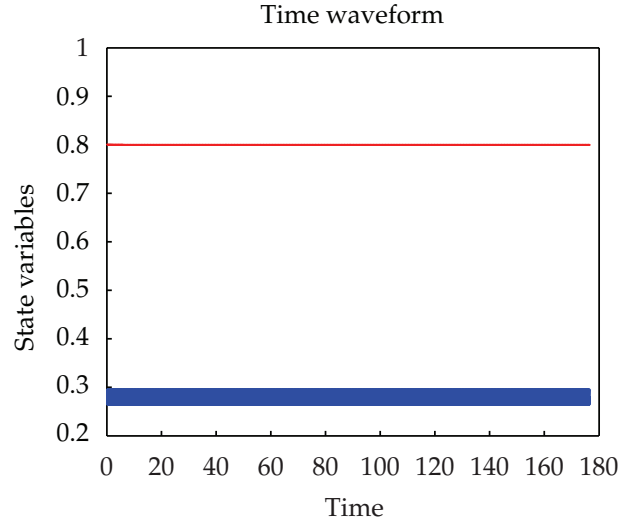

(b) Time waveform

Figure 6: 2-ZAD strategy for $k_{s}=2.5$ and initial conditions close to the reference value. 


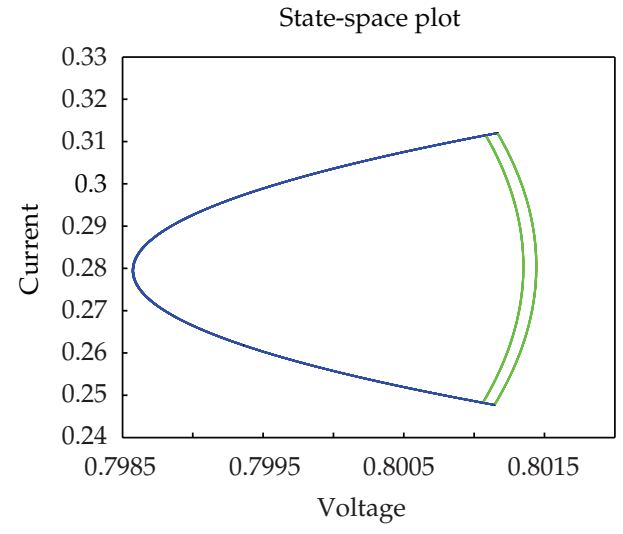

(a) Orbit in the state space

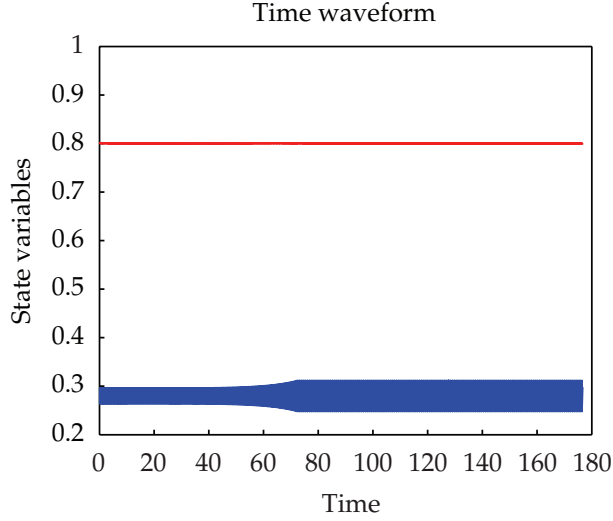

(b) Time waveform

Figure 7: Classical ZAD strategy for $k_{s}=2.5$ and initial conditions close to the reference value.

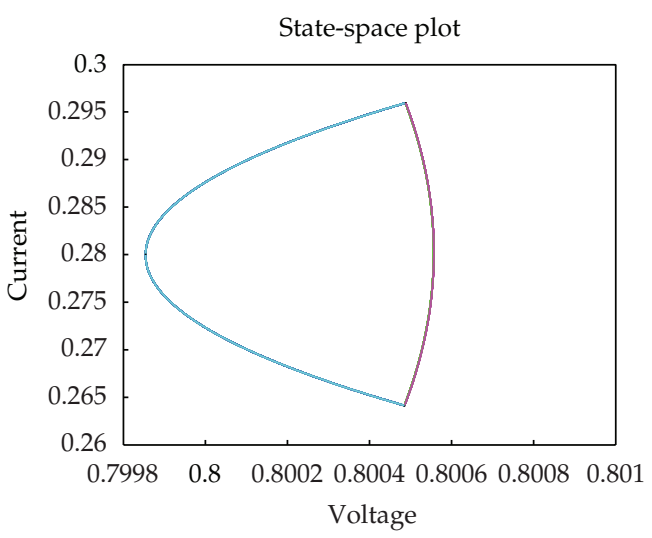

(a) Orbit in the state space

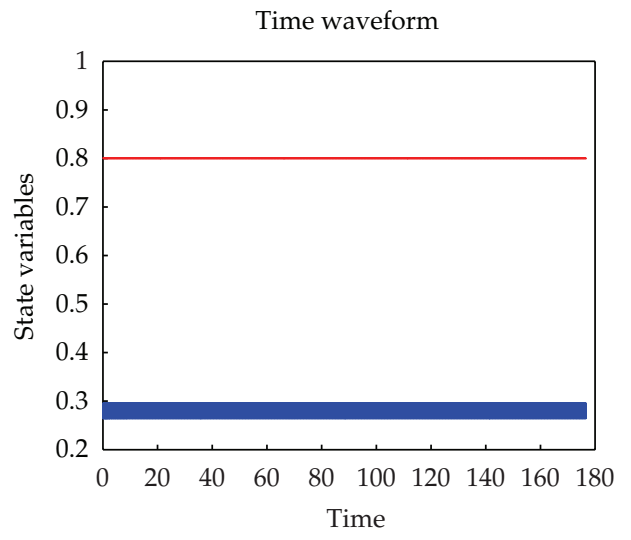

(b) Time waveform

Figure 8: 2-ZAD strategy for $k_{s}=1$ and initial conditions close to the reference value.

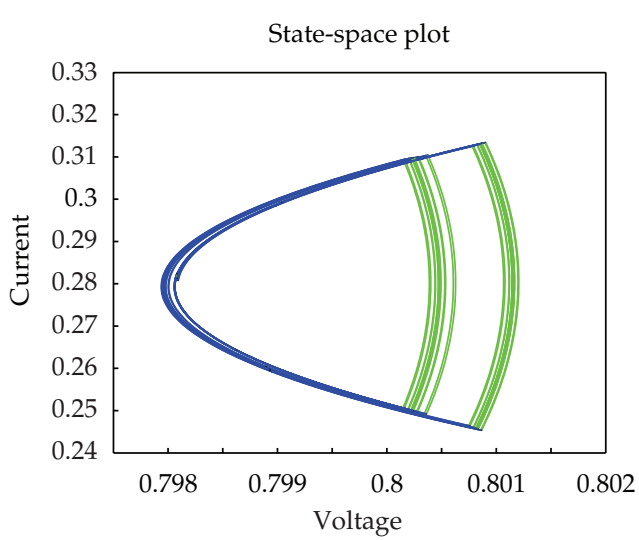

(a) Orbit in the state space

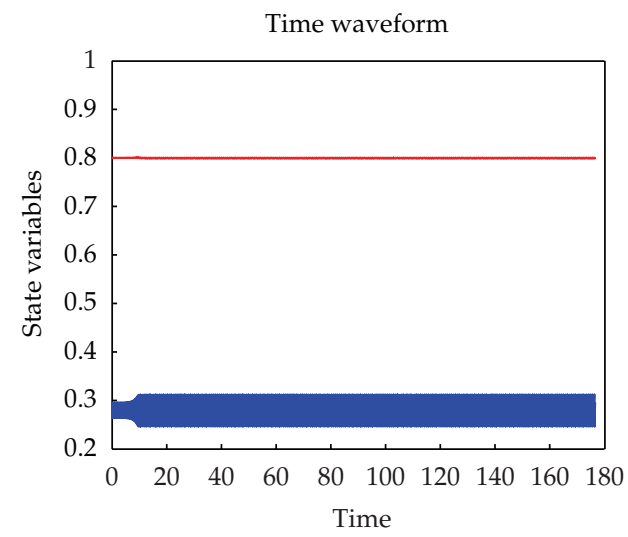

(b) Time waveform

Figure 9: Classical ZAD strategy for $k_{s}=1$ and initial conditions close to the reference value. 


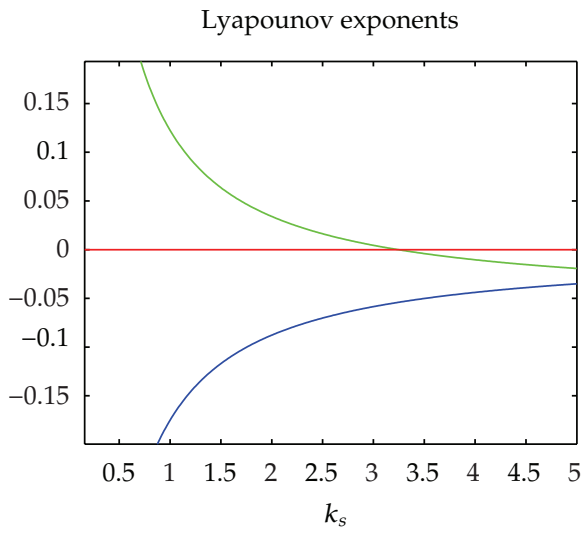

(a) Lyapunov exponents for the classical ZAD technique

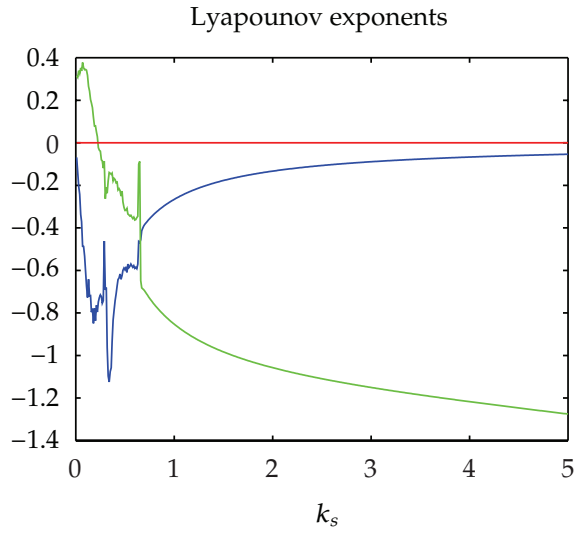

(b) Lyapunov exponents for 2-ZAD technique

Figure 10: Comparison of Lyapunov exponents.

\section{Conclusions and Future Work}

A generalization of the so-called ZAD technique (named $K$-ZAD) was described. It can be used as an alternative to classical ZAD when saturation effects lead the system to big amplitude chaotic behavior. In fact, 2-ZAD shows better performance than classical ZAD in all cases (regarding stability and nonsaturation). Even it can be used without additional stabilization methods such as FPIC or TDAS. Thus the control algorithm becomes simpler. Good performance of $K-Z A D$ is mainly dependent on the way that the (independent) duty cycles are computed. Several criteria can be chosen. The one chosen in this paper showed very good success. But it is worth to note that different criteria will lead to probably very different dynamics. Thus even better results than those shown in this paper can be obtained.

It is expected that as the value of $K$ is increased, better results can be obtained. But also it is evident that the control algorithm gets more complex. Some early results with 3ZAD (not shown in this paper) confirm this last sentence. Anyway, trying values bigger than 2 with some sort of optimal condition is proposed as our next step into further understanding the ZAD strategy.

\section{Acknowledgments}

The authors acknowledge partial financial support to CeiBA Complexity and to the prject Dima-vicerrectoría de investigación no. 20201006570 "Cálculo Científico para Procesamiento Avanzado de Bioseñales".

\section{References}

[1] L. O. Chua, "Special issue on chaos in electronic systems; tutorial and descriptive articles for the non-specialist," Proceedings of the IEEE, vol. 75, no. 8, 1987.

[2] J. Baillieul, R. W. Brockett, and R. B. Washburn, "Chaotic motion in nonlinear feedback systems," IEEE Transactions on Circuits and Systems, vol. 27, no. 11, pp. 990-997, 1980. 
[3] J. H. B. Deane and D. C. Hamill, "Instability, subharmonics and chaos in power electronic systems," in Proceedings of the 20th Annual IEEE Power Electronics Specialists Conference (PESC'89), pp. 34-42, June 1989.

[4] J. H. B. Deane and D. C. Hamill, "Analysis, simulation and experimental study of chaos in the buck converter," in Proceedings of the 21st Annual IEEE Power Electronics Specialists Conference (PESC '90), pp. 491-498, June 1990.

[5] P. T. Krein and R. M. Bass, "Multiple limit cycle phenomena in switching power converters," in Proceedings of the 4th Annual IEEE Applied Power Electronics Conference and Exposition (APEC '89), pp. 143-148, March 1989.

[6] D. Hamill, "Power electronics: a field rich in nonlinear dynamics," in Workshop on Nonlinear Dynamics of Electronic Systems, pp. 164-179, 1995.

[7] J. R. Wood, "Chaos: a real phenomenon in power electronics," in Proceedings of the 4th Annual IEEE Applied Power Electronics Conference and Exposition (APEC '89), pp. 115-124, March 1989.

[8] E. Ott, C. Grebogi, and J. A. Yorke, "Controlling chaos," Physical Review Letters, vol. 64, no. 11, pp. 1196-1199, 1990.

[9] K. Pyragas, "Continuous control of chaos by self-controlling feedback," Physics Letters A, vol. 170, no. 6, pp. 421-428, 1992.

[10] V. I. Utkin, "Variable structure systems with sliding modes," IEEE Transactions on Automatic Control, vol. 22, no. 2, pp. 212-222, 1977.

[11] V. I. Utkin, Sliding Modes and Their Applications in Variable Structure Systems, Mir, Moscow, Russia, 1978.

[12] M. Carpita, M. Marchesoni, M. Oberti, and L. Puguisi, "Power conditioning system using sliding mode control," in Proceedings of the IEEE Power Electronics Specialist Conference, pp. 623-633, 1988.

[13] F. Angulo, Análisis de la Dinámica de Convertidores Electrónicos de Potencia Usando PWM basado en Promediado Cero de la Dináamica del Error (ZAD) [Ph.D. thesis], Universidad Politéecnica de Cataluña, 2004.

[14] Y. A. Kuznetsov, Elements of Applied Bifurcation Theory, vol. 112 of Applied Mathematical Sciences, Springer, New York, NY, USA, 3rd edition, 2004. 


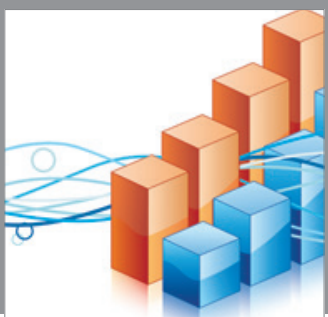

Advances in

Operations Research

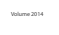

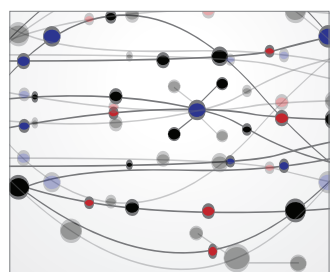

\section{The Scientific} World Journal
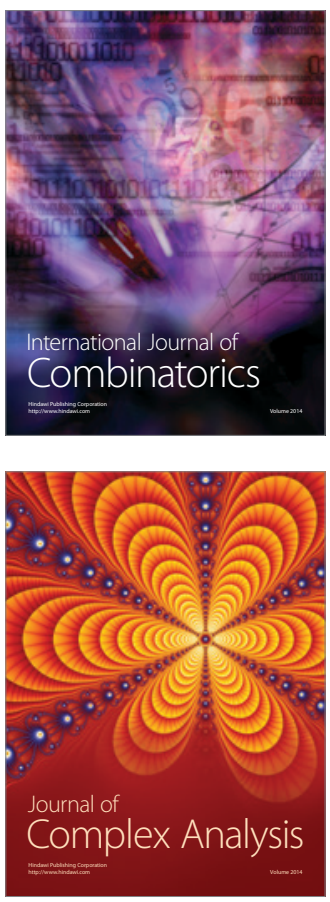

International Journal of

Mathematics and

Mathematical

Sciences
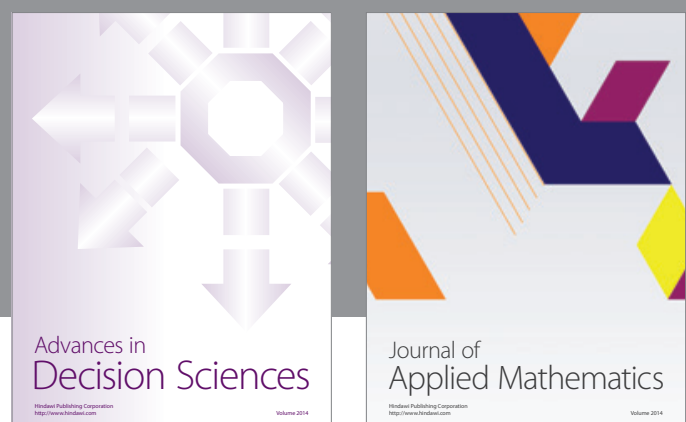

Journal of

Applied Mathematics
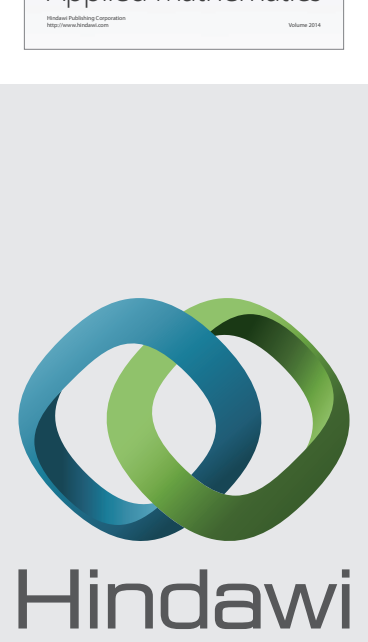

Submit your manuscripts at http://www.hindawi.com
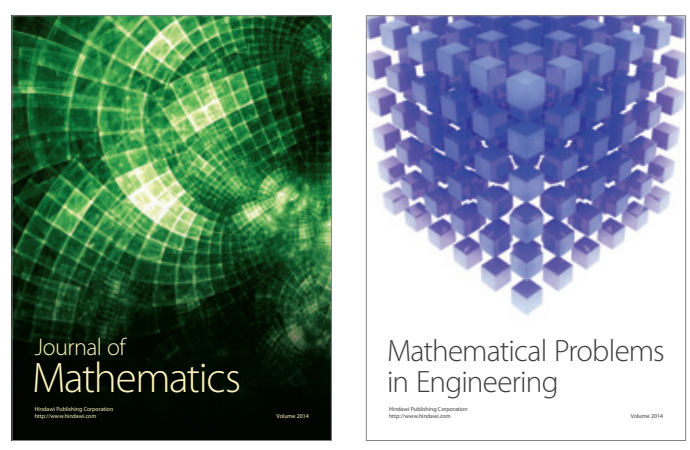

Mathematical Problems in Engineering
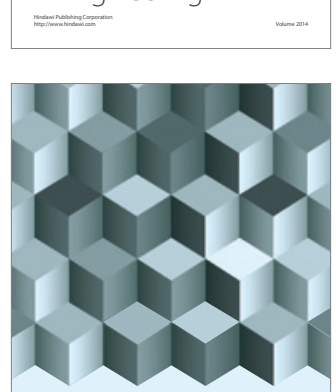

Journal of

Function Spaces
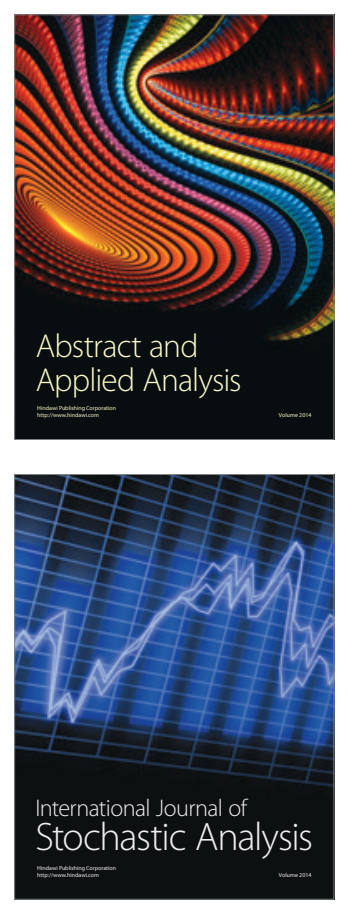

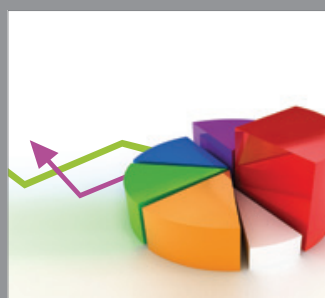

ournal of

Probability and Statistics

Promensencen
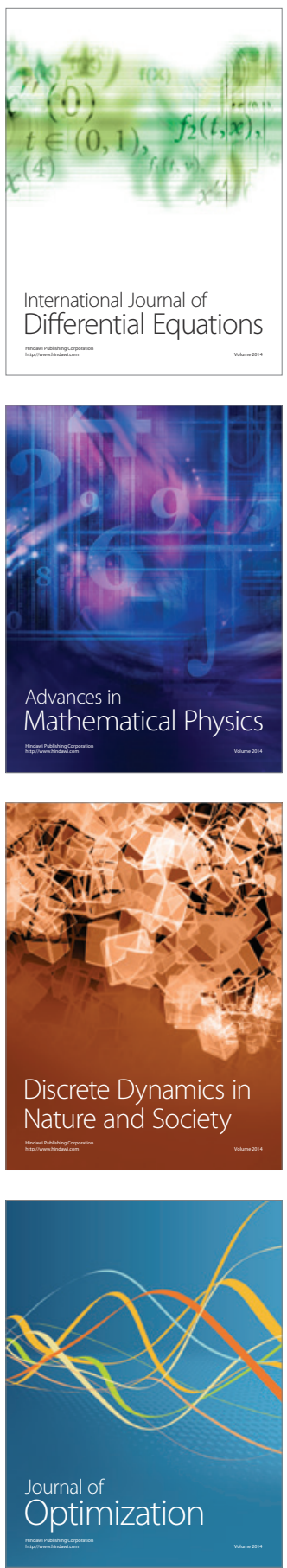